\title{
Hyaluronan, the backbone of perineuronal nets, supports glutamate transporter activity
}

Mariko Hayashi ${ }^{1,2}$, Kaoru Sato ${ }^{2}$

${ }^{I}$ Medical School, International University of Health and Welfare, Japan, ${ }^{2}$ National Institute of Health Sciences, Japan

Perineuronal nets are extracellular matrix structure formed around neuronal soma and dendrites. Their abnormality, as well as imbalance in excitatory and inhibitory circuits, underlies psychiatric diseases such as schizophrenia or autism spectrum disorders. However, little was known about how perineuronal nets affect neuronal activity. Here we show that hyaluronan, the backbone of perineuronal nets, supports glutamate transporter GLT1 to turn off excitatory signals. GLT1 lost glutamate uptake activity and retracted from cellular processes upon hyaluronidase treatment. Hyaluronan synthase HAS3, a transmembrane protein that synthesizes, secretes and anchors hyaluronan at the plasma membrane, interacted with GLT1 in a hyaluronan dependent manner. All three subtypes of hyaluronan synthases were capable of recruiting GLT1 to cellular processes. Hyaluronidase treatment activated neurons through glutamate receptors, and its prolonged treatment resulted in neuronal damage. Our work reveals how perineuronal nets spatiotemporally restricts excitatory synaptic transmission for healthy tuning of excitatory and inhibitory balance. 